\title{
Sebaceoma of a Meibomian Gland of the Upper Eyelid
}

\author{
Frederick A. Jakobiec ${ }^{\text {a }}$ Paula Cortes Barrantes ${ }^{\text {a }}$ Tatyana Milman $^{\text {b }}$ \\ Michael Yoon ${ }^{c}$
}

${ }^{a}$ David G. Cogan Laboratory of Ophthalmic Pathology, Massachusetts Eye and Ear/Harvard Medical School, Boston, MA, USA; b Department of Ophthalmic Pathology, Wills Eye Hospital, Sidney Kimmel Medical College of Thomas Jefferson University, Philadelphia, PA, USA; 'Department of Oculoplastics, Massachusetts Eye and Ear/ Harvard Medical School, Boston, MA, USA

\section{Established Facts}

- Sebaceoma is a rare tumor of the eyelid and periocular skin.

- Basaloid cells should constitute at least $50 \%$ of the cells in solid lesions, in contrast to the more differentiated sebaceous adenoma, which has a single outer layer of basaloid cells in a regular lobular architecture.

- Like sebaceous adenoma, sebaceoma may be associated with the Muir-Torre syndrome.

\section{Novel Insights}

- Sebaceomas may arise in the Meibomian glands, as it did in a 74-year-old man.

- The morphology of this tumor can be highly variegated, with interconnecting cords of haphazardly arranged basaloid and sebaceous cells (sebocytes) in a cystic cavity, as in the present case.

- In the current case, both a positive family history and mismatch repair defects in the patient's protein expression for MLH1 and PMS2 were factors, indicating that eyelid sebaceoma can potentially be associated with the Muir-Torre syndrome.

\section{Keywords}

Sebaceoma $\cdot$ Sebaceous adenoma $\cdot$ Sebaceous hyperplasia $\cdot$ Sebaceous carcinoma $\cdot$ Muir-Torre syndrome $\cdot$ Mismatch repair proteins $\cdot$ Eyelid $\cdot$ Meibomian gland

\section{Abstract \\ Over a period of 1 year, a 74-year-old man slowly developed a painless left upper eyelid intratarsal mass. The skin was movable over the lesion. At surgery, a well-circumscribed, yellow-white, partially cystic tumor was encountered. Histo-}

(c) 2020 S. Karger AG, Basel

www.karger.com/oop

Karger ${ }^{\prime}=$ pathologically it was composed of a random mixture of basaloid and sebaceous cells arranged in interconnecting cords. Immunohistochemical evaluation disclosed epithelial membrane antigen, adipophilin, and cytokeratin 14 positivity. These findings led to the diagnosis of a sebaceoma. The tumor cells abnormally failed to express mismatch repair proteins for MLH1 and PMS2. The patient did not have a personal history of any visceral malignancy, but his father had died at the age of 46 years and a daughter at the age of 33 years from colonic carcinomas. The implications of this periocular sebaceoma for the Muir-Torre syndrome are explored.

(c) 2020 S. Karger AG, Base

Frederick A. Jakobiec, MD, DSc

David G. Cogan Laboratory of Ophthalmic Pathology

Massachusetts Eye and Ear Infirmary, 243 Charles Street, Suite 328

Boston, MA 02114 (USA)

E-Mail Fred_Jakobiec@meei.harvard.edu 


\section{Introduction}

Sebaceoma is a tumor situated nosologically between a sebaceous adenoma and a sebaceous carcinoma [1-4]. It is not as highly organized as a pseudoadenomatous sebaceous gland hyperplasia or an adenoma, lacking their preserved lobular architecture [5]. Care must be taken to distinguish a sebaceoma from a well-differentiated sebaceous carcinoma. Sebaceomas are infrequently diagnosed lesions of the ocular adnexa, and to the best of our knowledge, no case has yet been reported to have originated in a Meibomian gland. Like sebaceous adenoma [6], sebaceoma is a potential harbinger of the Muir-Torre syndrome (MTS) [7], which is characterized by several types of visceral malignancies [8-14].

Sebaceomas usually develop in the scalp or on the face [15]; the periocular tissues appear to be an extremely rare site of origin. There are two reports of a sebaceoma of the eyelid margin $[16,17]$, and both were unassociated with the MTS. We describe for the first time a sebaceoma located in the tarsus that developed from a Meibomian gland. The lesion was evaluated immunohistochemically and found to have DNA mismatch repair (MMR) protein expression defects. A positive family history of colonic carcinomas at an early age provided further evidence for the likelihood of the MTS.

\section{Case Report}

A 74-year-old man with a personal history of facial squamous cell carcinoma presented for evaluation of a left upper eyelid lesion. It had been present for approximately 1 year. He reported occasional blurring of vision without pain, swelling, redness, or discharge. There was no history of trauma. He divulged no personal history of any visceral malignancy. There was a family history of intestinal cancer: his father had died at age 46 from colonic carcinoma, and a daughter had died at age 33 from the same disease; however, our patient had never undergone colonoscopy.

On ophthalmic examination, he had a $15 \times 10 \mathrm{~mm}$ lesion of the central left upper eyelid (Fig. 1a). There were no superficial skin abnormalities. The skin moved over the lesion, revealing a subcutaneous location. Eversion of the upper eyelid revealed a normalappearing tarsus with a smooth, non-ulcerated surface that was mildly elevated. An excision was performed through a skin crease. Blunt dissection through the orbicularis oculi muscle led to the mass that was situated in the tarsus and protruded beyond it (Fig. 1b). There was a smooth white surface capsule that blended with the tarsus and adhered to the superficial tissue.

A gross total excision was successfully achieved with preservation of the capsule. The patient has not experienced any recurrence during 9 months of follow-up.

\section{Results}

Histopathologic and Immunohistochemical Findings

At gross examination, the lesion was fluctuant, round, and tan-white with a white capsule and measured $1.3 \times$ $0.9 \times 0.6 \mathrm{~cm}$. On the cut surface, it was found to be yellowwhite and partially cystic. Microscopically, it was well circumscribed and composed of interconnecting cords of cells suspended in a background of eosinophilic amorphous material (holocrine secretion) (Fig. 1c). The cords displayed rounded collections of clear sebocytes enveloped by more numerous small germinal basaloid cells (Fig. 1d). Basaloid cells constituted at least $50 \%$ of the tumor's overall population (Fig. 1e). Some areas of the lesion were entirely bereft of any cellular clusters and instead only exhibited background eosinophilic material (Fig. 1f). Some of this material had leaked into the surrounding connective tissues and provoked a lipogranulomatous response (Fig. 1f, 2a).

Immunohistochemical staining with CAM 5.2 was positive only in the outer basaloid cells, while the sebocytes were negative (Fig. 2b). Adipophilin in a vesicular form was positive in many dispersed sebocytes (Fig. 2c). Cytokeratin 14 was uniformly positive in all of the cells comprising the inter-anastomosing cords (Fig. 2d). Epithelial membrane antigen was positive in small clusters of cells (Fig. 2e). The Ki-67 proliferation index was around $50 \%$ and restricted to the basal cells (Fig. 2f). Immunostaining for DNA MMR protein expression showed loss of MLH1 (Fig. 3a) and PMS2 (Fig. 3d), with preservation of MSH2 (Fig. 3b) and MSH6 (Fig. 3c). At a consensus conference in the Dermatopathology Division of the Department of Pathology at the Massachusetts General Hospital, the case was reviewed, discussed, and diagnosed as a sebaceoma.

\section{Discussion}

In a 1956 series [18] of 21 sebaceous tumors of all kinds developing in the Meibomian glands, there were 16 carcinomas, 3 adenomas, 1 hyperplasia, and 1 hamartoma. No mention of a sebaceoma was made, because the criteria for the identification of this entity were not codified until 1984 [19]. Since its characterization, it has emerged that it is a very rare lesion. In a series of 207 benign and malignant sebaceous tumors, only $1.6 \%$ were sebaceomas [20]. Before the diagnostic category of sebaceoma had been identified, sebaceomas were often called "sebaceous epitheliomas" [1, 19]. This term, however, created con- 

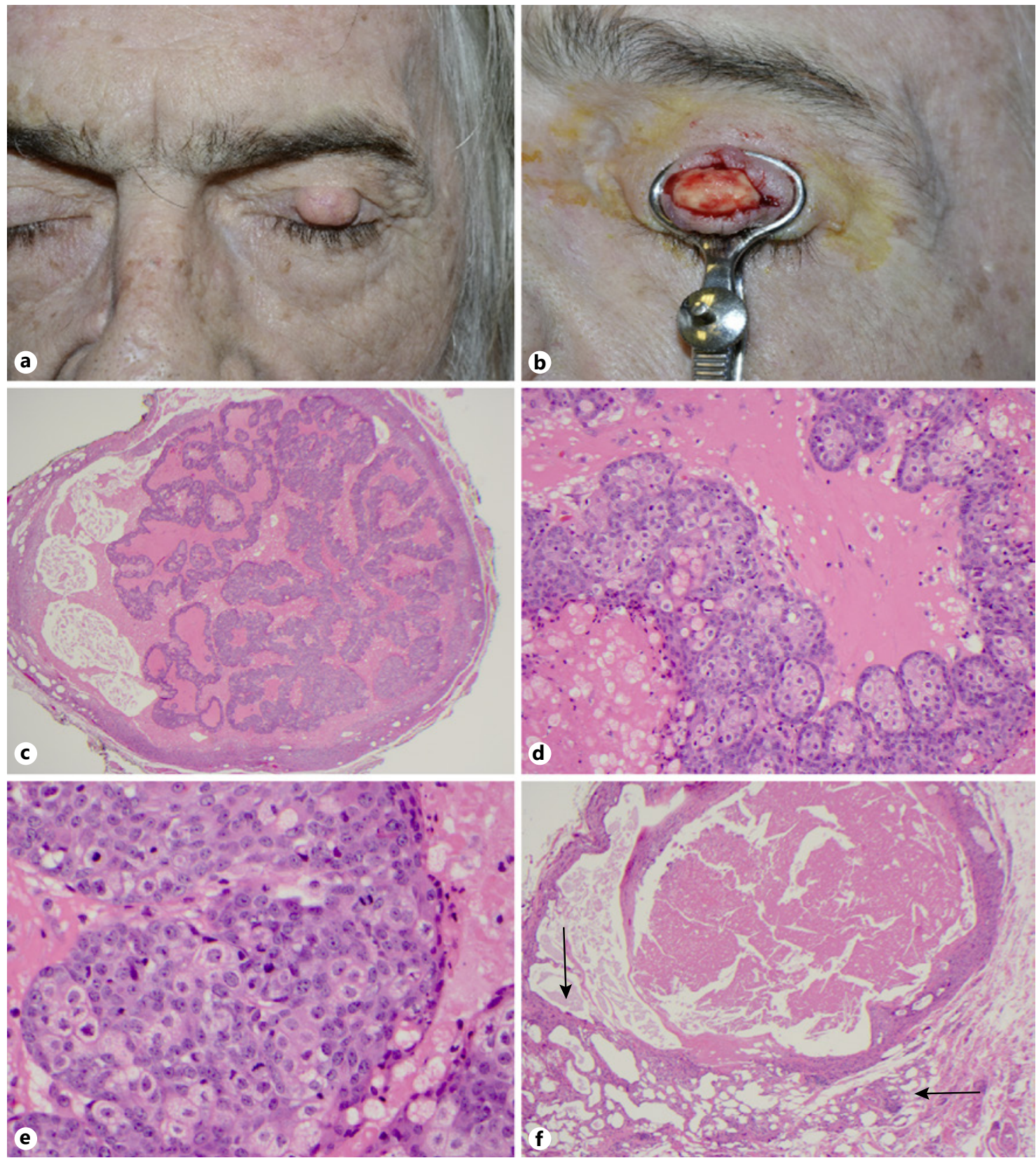

Fig. 1. Clinical and histopathologic features of a Meibomian gland sebaceoma. a A left mid-upper eyelid mass deep in the skin is brought out on downward gaze. $\mathbf{b}$ Smooth appearance of the blanched tarsal conjunctival surface, with a clamp everting the upper eyelid. c A well-circumscribed mass with interconnecting cellular cords is situated within a "cystic" background composed of amorphous eosinophilic material. H\&E. $\times 2$. $\mathbf{d}$ The cords are composed of clear cells and outer basaloid cells. H\&E. $\times 20$. e A higher-power image of the cellular composition of the cords reveals the random admixture of clear, vacuolated cells and smaller basaloid cells. H\&E. $\times 40 . \mathbf{f}$ There is a cell-free zone of the cyst's eosinophilic material that has escaped into the surrounding connective tissue to elicit a lipogranuloma (arrows). H\&E. $\times 4$. 

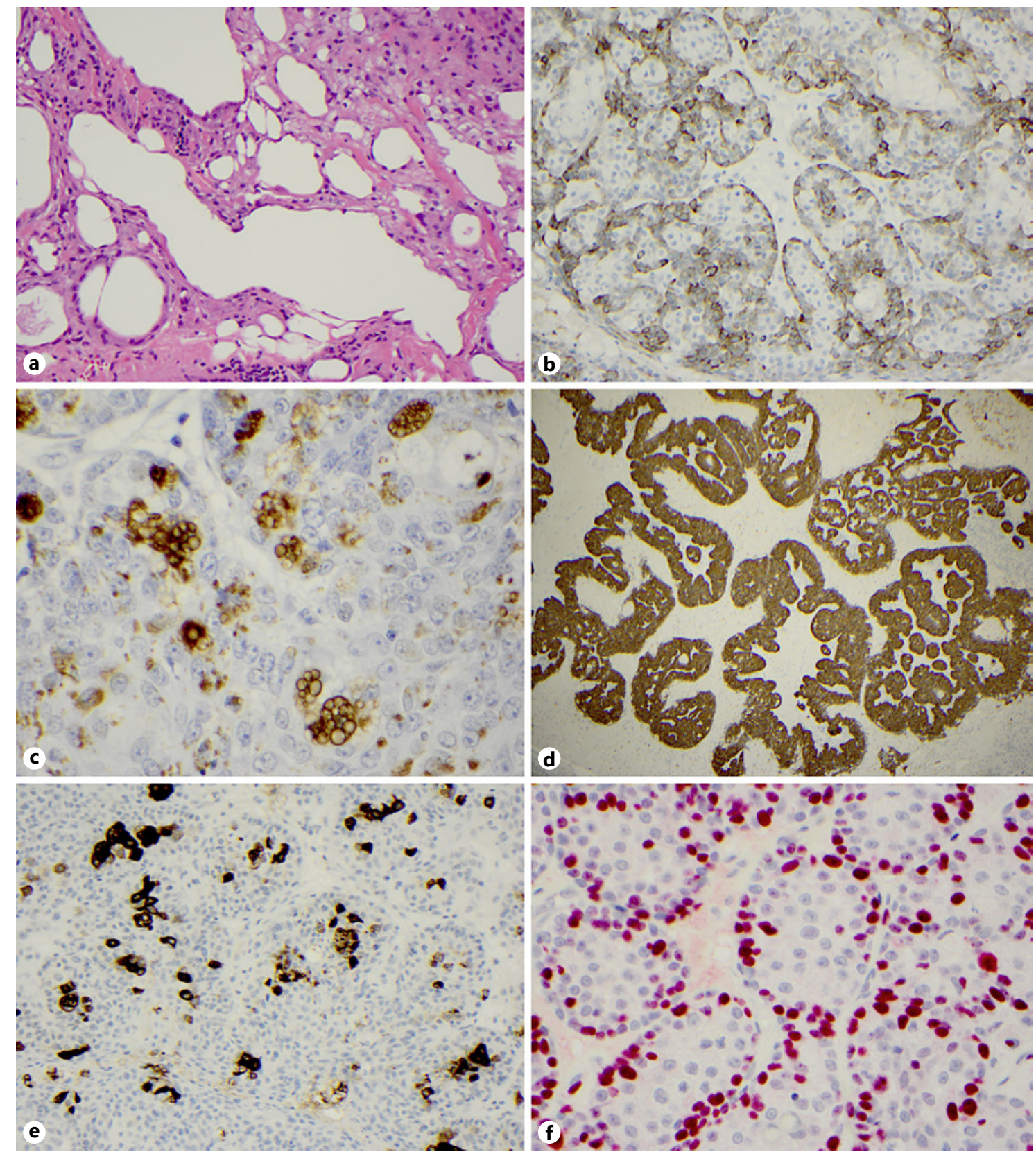

Fig. 2. Lipogranuloma and results of immunohistochemical biomarker studies. a Variously sized, dissolved-out extracellular lipid locules are surrounded by epithelioid cells. H\&E. $\times 20$. b CAM 5.2 cytokeratin staining reveals positivity only in outer basaloid cells. Immunoperoxidase, diaminobenzidine [DAB]-chromogen with hematoxylin counterstain. $\times 20$. c Adipophilin is positive in the highly diagnostic vesicular form in vacuolated sebocytes. Immunoperoxidase, DAB-chromogen with hematoxylin counterstain. $\times 60$. d Cytokeratin 14 uniformly stains all of the cells in the interconnecting cords. Immunoperoxidase, DAB-chromogen with hematoxylin counterstain. $\times 4$. e Epithelial membrane antigen is positive in scattered partially lipidized sebocytes. Immunoperoxidase, DAB-chromogen with hematoxylin counterstain. $\times 20$. f Ki-67 positivity is high among outer germinal basal cells (a proliferation index approaching 40\%). Immunoperoxidase, DAB-chromogen with hematoxylin counterstain. $\times 40$. 


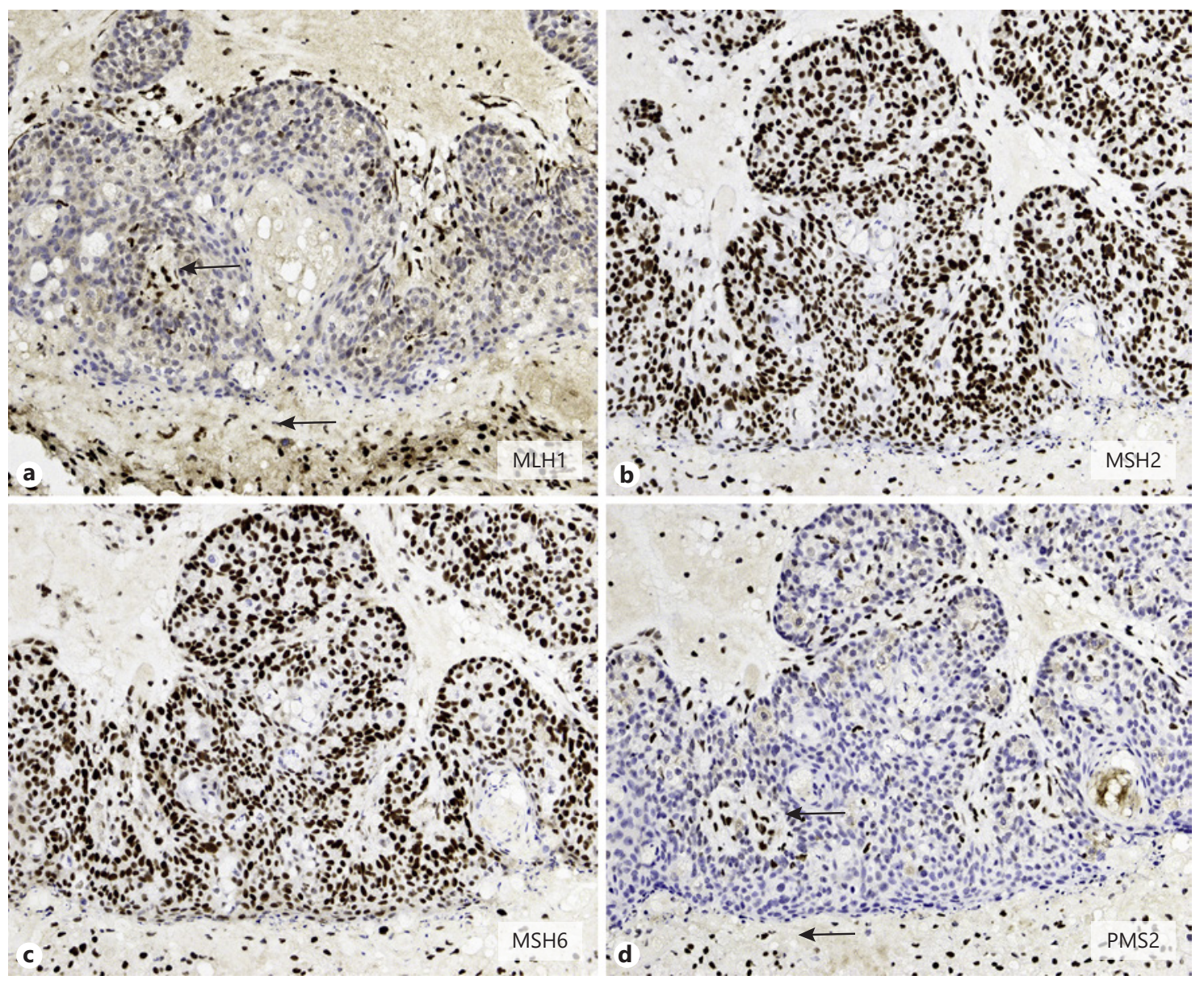

Fig. 3. Mismatch repair (MMR) nuclear protein immunogenetic studies. a MMR MLH1 nuclear protein is not expressed in the outer basaloid cells and inner vacuolated sebocytes (arrows). Immunoperoxidase, diaminobenzidine [DAB]-chromogen with hematoxylin counterstain. $\times 10$. b Preserved nuclear MSH2 protein expression is highlighted with brown (DAB) chromogen. Immuno- peroxidase, DAB-chromogen with hematoxylin counterstain. $\times 10$. c All cells express MMR MSH6 nuclear protein. Immunoperoxidase, DAB-chromogen with hematoxylin counterstain. $\times 10$. d Outer basaloid cells, inner intermediate cells, and sebocytes have lost expression of MMR PMS2 nuclear protein (arrows). Immunoperoxidase, DAB-chromogen with hematoxylin counterstain. $\times 10$. siderable confusion, since it was applied to benign tumors but sometimes also to some malignant tumors [19]. The malignant tumors formerly called "epitheliomas" are now referred to as "carcinomas."

Correctly diagnosed according to the initial criteria that were laid down [19], sebaceoma is a tumor of the adnexal epithelium rather than of the surface epithelium (epidermis) $[1,13,19]$. There is a predilection for siting in the head and neck region $[1,2,15]$; the eyelids and ears are generally spared. It is a larger tumor than the typical adenoma; it is well circumscribed but non-encapsulated and may rarely be multinodular. The organization of its cellular elements is what allows its separation from an adenoma. It is typically a well-demarcated yellow nodule, over which the epidermis may occasionally be hyperkeratotic or even verrucoid and, internally, the tumor may be partially cystic.

Only two previous reports allegedly describing a sebaceoma have appeared in the eye literature $[16,17]$, and both involved the eyelid margins. The first report [16] concerned a 53-year-old man who over 2 months had developed a left lower eyelid margin tumor that was entirely situated in the dermis. It arose either from a Zeis gland or a Meibomian gland lobule (alveolus) located near the ductal orifice. The patient had a loss of DNA MMR protein expression for MMR MSH2 and MSH6, despite the fact that there was no family or personal history of visceral malignancy. The second case [17] occurred in an 
81-year-old man with a marginal right upper eyelid nodule that had appeared over a 5-month period. Microscopically, it arose from the epidermis, and therefore not from the adnexal epithelium (the follicular-apocrine-sebaceous complex). Its origin was multifocal, from the epidermis, with focal sebocytic differentiation and peripheral palisading. This tumor, in retrospect, is more consistent with a basal cell carcinoma displaying sebaceous differentiation. The expression of MMR protein MSH6 was absent, yet the patient and his family were not known to have any internal malignancies. A basal cell carcinoma with focal sebaceous differentiation is generally not considered to be a lesion associated with the MTS [21].

The cardinal histopathologic feature of a sebaceoma is that of randomly or haphazardly distributed single sebocytes or small clusters of these cells in a background composed predominantly (more than 50\%) of basaloid and/ or intermediate or transitional cells $[1-4,16]$. The latter are basaloid in type but display more ample cytoplasm than classic basaloid cells and may also exhibit early subtle cytoplasmic lipidization. Rare duct-like structures and cystification can occasionally be observed. This lesion, consequently, differs from a sebaceous adenoma $[6,18]$, which manifests an organized lobular architecture with one or two layers of germinal basaloid cells at the periphery of the lobules and fully lipidized central sebocytes undergoing holocrine dissolution. Sebaceoma, unlike sebaceous adenoma, may show varying degrees of nuclear atypia and an elevated mitotic index, features that do not imply malignancy.

Immunohistochemistry can play a valuable adjunctive role in diagnosis. Cytokeratins identified with CAM 5.2 and cytokeratin 14 were all positive in the sebaceoma, along with focal positivity for epithelial membrane antigen. Most important is the identification of cytoplasmic lipidic inclusions in paraffin sections stained with adipophilin [22-24]. Inclusions with a vesicular appearance are specific for endogenous lipid synthesis and were seen in the current lesion. Granular inclusions are nonspecific and can be secondary degenerative phenomena or the result of phagocytosis of lipidic debris from nearby necrotic cells.

The fundamental biologic importance of sebaceomas, shared with sebaceous adenomas, is their possible association with the MTS [7-14], an autosomal dominant condition predisposing to one or more internal visceral malignancies, most often of the colon, but also of the lung, stomach, endometrium, urothelium, and skin. Around $15 \%$ of colonic cancers are linked to the MTS. DNA MMR protein defects are usually, but not always, found in the tumor cells' nuclei in MTS-associated skin neoplasms. This association has not been displayed by pseudoadenomatous hyperplasia of sebaceous glands [5].

Double allelic germline mutations underwrite the MTS. One allelic mutation may be followed by a second allelic "hit" (Knudson hypothesis) [7]. Immunogenetic examination of the tumor is performed to detect the absence of a nuclear protein expression in one of the major DNA MMR genes - MLH1, MSH2, PMS2, and MSH6. In the current case, such defective nuclear protein expression was discovered for MLH1 and PMS2. The patient has not yet developed any evidence of the MTS in terms of visceral malignancy, and despite a strong family history of early-onset colon carcinoma, he has declined a thorough systemic workup including a colonoscopy. It is conceivable that the patient has escaped a germline mutation and that his MMR defective expression is a result of a somatic mutation restricted to his tumor. It has been known for some time that MMR defects from a clinical perspective are not totally reliable in the detection of the MTS [25]. Nonetheless, the overwhelming evidence strongly suggests that our patient fits the category of the MTS.

A paper in 2008 [15] provided some very interesting data on the location of sebaceomas and adenomas with regard to the development of the MTS. Ninety-two benign and malignant sebaceous tumors of all types were studied, revealing that $49 \%$ were sebaceous adenomas, $37 \%$ sebaceous carcinomas, and $14 \%$ sebaceomas. Overall, there were 79 tumors with known sites. $82 \%$ ( 39 of 47 ) arose in the head and neck region. Interestingly, when it came to the carcinomas, $63 \%$ (22 of 35) were located in the non-head and neck region. MMR protein deficiency was found in $92 \%$ of the non-head and neck tumors, but in only $27 \%$ of the head and neck tumors. Of particular interest is that only $7 \%$ of the periocular sebaceous tumors had an MMR deficiency. In analyzing the full extent of involved sites, $45 \%$ of the benign tumors had MMR defects, whereas only $15 \%$ of the carcinomas did.

It was once believed that cystic or hyperkeratotic (verrucoid) sebaceomas had a higher likelihood of association with the MTS, but this has definitely been disproved [15]. Furthermore, not all sebaceomas have a strong linkage with the MTS. There is a group of morphologically distinctive sebaceomas that display variant morphologies and constitute a distinct minority not usually associated with the MTS. This subset of lesions has been called "patterned" sebaceomas, which have a tendency to involve the scalp of male patients. In a group of 31 patients with these lesions with clinical follow-ups, only 5 developed an internal malignancy [26]. 
Only recently [26] has this group of patterned lesions been microscopically described in the literature. In brief, they exhibit cigar-shaped cells that assume mixed patterns combining a full range of morphologic expressions. The four fundamental patterns are: (1) carcinoid-like (ribbons or trabeculae); (2) labyrinthine/sinusoidal (cords and strands separated by stroma); (3) rippled (tightly regimented, non-lumen-forming cells in palisades or forming Verocay body-like structures); and (4) petaloid (twisted floral-like cellular arrangement). Occasionally, duct-like structures can be seen. Patterned sebaceomas have not yet been described in the periocular skin.

The management of sebaceoma consists of a wider local excision than that performed for a straightforward adenoma, because of its larger size and higher mitotic index. However, the lesion rarely recurs and does not metastasize. A thorough personal and family clinical history must be taken from the patient to establish any evidence of a familial proneness to internal malignancies (colon, lung, endometrium, etc.). Any organ symptoms at the time of a patient's presentation with a sebaceoma should raise suspicion about a possible occult malignancy and should be followed up immediately with appropriate testing, including colonoscopy.

All resected tumors should be evaluated for lack of expression of a DNA MMR nuclear protein as a screening strategy for highlighting the possibility of the MTS. As pointed out above [15], benign and malignant periocular sebaceous neoplasms have a lower yield when tested for a defect in MMR protein expression. We discovered loss of MLH1 and PMS2 protein expression in the current neoplasm. This expression pattern is unusual, since the MTS is more frequently associated with loss of $\mathrm{MSH} 2$ and MSH6 expression and an underlying $\mathrm{MSH} 2$ gene mutation. Not all sebaceomas associated with the MTS, however, harbor an MMR defect [25].

MLH1 and PMS2 germline mutations have also been identified in the MTS and may display regional variation. The loss of MLH1 and PMS2 expression does raise a question regarding the possibility of somatic MLH1 promoter hypermethylation [27], a known mechanism for neoplasia in non-cutaneous malignancies, such as colorectal carcinoma and uterine adenocarcinoma. This mechanism in sebaceous neoplasms with MLH1 protein expression loss has not been formally explored.

In summary, our patient had no personal but a positive family history of MTS-associated tumors appearing at an early age and a somatic mutation in MLH1 or PMS2. The mutation could have been mediated by hypermethylation rather than a germline mutation. This is only an interest- ing hypothesis to explain the observed immunohistochemical findings and lack of visceral malignancy in the present patient. A germline mutation can be reliably identified only by studying peripheral blood cells or normal non-tumoral tissue. This is an expensive methodology that may not be covered by many insurance policies. This test was not performed on our patient, who rejected any thoroughgoing workup.

\section{Statement of Ethics}

This study complies with the tenets of the Declaration of Helsinki and Health Insurance Portability and Accountability Act regulations. The clinical photographs have been cropped to protect the patient's identity. Nonetheless, all surgical consent forms signed by the patient at our institution contain an approval for the clinical photographs to be used in any future publications.

\section{Disclosure Statement}

The authors have no financial or proprietary interests in the materials mentioned herein. No conflicting relations exist for any author.

\section{Funding Sources}

Department of Ophthalmology Research Fund, Massachusetts Eye and Ear. This research did not receive any specific grant from funding agencies in the public, commercial, or non-profit sectors.

\section{Author Contributions}

Frederick A. Jakobiec: main author; responsible for supervision, conceptual guidance, and writing of the manuscript. Paula Cortes Barrantes: responsible for data summary, preparation, library search, references, and illustrations. Tatyana Milman: responsible for additional MMR immunohistochemical staining and interpretation of the results; consultant for the genetic and molecular concepts and interpretations. Michael Yoon: provided the case report and patient follow-up.

References

1 Elder DE, Elenitsas R, Murphy G, Xu X. Benign pigmented lesions and malignant melanoma. In: Elder DE, Elenitsas R, Rosenbach M, editors. Lever's histopathology of the skin. 11th ed. Philadelphia: Wolters Kluwer; 2015. pp. 872-3.

2 Weedon D. Tumors of cutaneous appendages. In: Weedon D, editor. Weedon's skin pathology. 3rd ed. China: Churchill-Livingstone; 2010. p. 773-4. 
3 Iacobelli J, Harvey NT, Wood BA. Sebaceous lesions of the skin. Pathology. 2017 Dec;49(7): 688-97.

4 Flux K. Sebaceous neoplasms. Surg Pathol Clin. 2017 Jun; 10(2):367-82.

5 Jakobiec FA, Cortes Barrantes P, Milman T, Lee NG, Fay A. Ocular adnexal adenomatoid sebaceous gland hyperplasia: a clinical and immunopathologic analysis in relation to the Muir-Torre syndrome. Ophthal Plast Reconstr Surg. 2020 Jan/Feb;36(1):e6-12.

6 Jakobiec FA. Sebaceous adenoma of the eyelid and visceral malignancy. Am J Ophthalmol. 1974 Dec;78(6):952-60.

7 Milman T, McCormick SA. The molecular genetics of eyelid tumors: recent advances and future directions. Graefes Arch Clin Exp Ophthalmol. 2013 Feb;251(2):419-33.

8 Carethers JM, Stoffel EM. Lynch syndrome and Lynch syndrome mimics: the growing complex landscape of hereditary colon cancer. World J Gastroenterol. 2015 Aug;21(31) 9253-61.

9 Jakobiec FA, Zimmerman LE, La Piana F, Hornblass A, Breffeilh RA, Lackey JK. Unusual eyelid tumors with sebaceous differentiation in the Muir-Torre syndrome. Rapid clinical regrowth and frank squamous transformation after biopsy. Ophthalmology. 1988 Nov;95(11):1543-8.

10 Haraldsdottir S, Hampel H, Tomsic J, Frankel WL, Pearlman R, de la Chapelle A, et al. Colon and endometrial cancers with mismatch repair deficiency can arise from somatic, rather than germline, mutations. Gastroenterology. 2014 Dec;147(6):1308-1316.e1.

11 Sourrouille I, Coulet F, Lefevre JH, Colas C, Eyries M, Svrcek M, et al. Somatic mosaicism and double somatic hits can lead to MSI colorectal tumors. Fam Cancer. 2013 Mar; 12(1):27-33.
12 Schon K, Rytina E, Drummond J, Simmonds J, Abbs S, Sandford R, et al. Evaluation of universal immunohistochemical screening of sebaceous neoplasms in a service setting. Clin Exp Dermatol. 2018 Jun;43(4):410-5.

13 Orta L, Klimstra DS, Qin J, Mecca P, Tang LH Busam KJ, et al. Towards identification of hereditary DNA mismatch repair deficiency: sebaceous neoplasm warrants routine immunohistochemical screening regardless of patient's age or other clinical characteristics. Am J Surg Pathol. 2009 Jun;33(6):934-44.

14 Boennelycke M, Thomsen BM, Holck S. Sebaceous neoplasms and the immunoprofile of mismatch-repair proteins as a screening target for syndromic cases. Pathol Res Pract. 2015 Jan;211(1):78-82.

15 Singh RS, Grayson W, Redston M, Diwan AH, Warneke CL, McKee PH, et al. Site and tumor type predicts DNA mismatch repair status in cutaneous sebaceous neoplasia. Am J Surg Pathol. 2008 Jun;32(6):936-42.

16 Yonekawa Y, Jakobiec FA, Zakka FR, Fay A. Sebaceoma of the eyelid. Ophthalmology. 2012 Dec;119(12):2645.e1-4.

17 Mittal R, Tripathy D. Sebaceoma of the eyelid: a rare entity. Can J Ophthalmol. 2014 Jun; 49(3):e78-80.

18 Straatsma BR. Meibomian gland tumors. AMA Arch Opthalmol. 1956 Jul;56(1):71-93.

19 Troy JL, Ackerman AB. Sebaceoma. A distinctive benign neoplasm of adnexal epithelium differentiating toward sebaceous cells. Am J Dermatopathol. 1984 Feb;6(1):7-13.
20 Manonukul J, Kajornvuthidej S. Sebaceous neoplasms in Siriraj Hospital, Mahidol University: a 9-year-retrospective study. J Med Assoc Thai. 2010 Aug;93(8):978-91.

21 Schwartz RA, Torre DP. The Muir-Torre syndrome: a 25-year retrospect. J Am Acad Dermatol. 1995 Jul;33(1):90-104.

22 Jakobiec FA, Mendoza PR. Eyelid sebaceous carcinoma: clinicopathologic and multiparametric immunohistochemical analysis that includes adipophilin. Am J Ophthalmol. 2014 Jan;157(1):186-208.e2.

23 Milman T, Schear MJ, Eagle RC Jr. Diagnostic utility of adipophilin immunostain in periocular carcinomas. Ophthalmology. 2014 Apr; 121(4):964-71.

24 Ostler DA, Prieto VG, Reed JA, Deavers MT, Lazar AJ, Ivan D. Adipophilin expression in sebaceous tumors and other cutaneous lesions with clear cell histology: an immunohistochemical study of 117 cases. Mod Pathol. 2010 Apr;23(4):567-73.

25 Ko CJ. Muir-Torre syndrome: facts and controversies. Clin Dermatol. 2010 May-Jun; 28(3):324-9.

26 Wiedemeyer K, Kyrpychova L, Işikci OT, Spagnolo DV, Kutzner H, Rütten A, et al. Sebaceous neoplasms with rippled, labyrinthine/sinusoidal, petaloid, and carcinoid-like patterns: a study of 57 cases validating their occurrence as a morphological spectrum and showing no significant association with MuirTorre syndrome or DNA mismatch repair protein deficiency. Am J Dermatopathol. 2018 Jul;40(7):479-85.

27 Newton K, Jorgensen NM, Wallace AJ, Buchanan DD, Lalloo F, McMahon RF, et al. Tumour MLH1 promoter region methylation testing is an effective prescreen for Lynch Syndrome (HNPCC). J Med Genet. 2014 Dec; 51(12):789-96. 